\title{
A time of reflection: a time for change
}

Jeff Moyer ${ }^{1}$

Accepted: 22 April 2020 / Published online: 12 May 2020

(c) Springer Nature B.V. 2020
The world has come to a screeching halt. A global pandemic has shaken the foundation of life as we know it, and things may never be the same. And that can and should be a catalyst for change. We are presented with a unique opportunity to look critically at many of our society's systems and not only challenge them but challenge ourselves. Societies are given few opportunities to reset their trajectory; we have been given such a chance. To simply suggest we return to "normal" will be to miss this opportunity.

COVID-19 has exposed the flaws and weaknesses in a broken food system that depends on high volumes of external inputs, long distance and international supply chains, and operates with a disregard for soil health and the health of those who consume the food. The health of people is intrinsically linked to the health of our soils.

In 1942, Rodale Institute founder J.I. Rodale wrote some words on a blackboard. He wrote: "Healthy Soil= Healthy Food = Healthy People," galvanizing the idea that our health and the health of soil are linked. Over the decades that have followed, science has proven this to be true (Steffan et al. 2018). Still, human health has not become a primary goal of agriculture. Our current food system is most adept at producing low-cost, highly processed, hyperpalatable, nutrient-poor foods and commodities like wheat, corn, soy, and rice, many of which end up as livestock feed, ingredients for non-food uses, or refined and processed foods. Agricultural intensification and consolidation have enabled the mass-scale production of inexpensive, low quality crop and animal products sacrificing our health.

Yet scientific research overwhelmingly supports the adoption of a diet high in nutrient-dense fruits, vegetables, grains, and livestock products (Alonso et al. 2019; Slavin and Lloyd 2012). Simply put, our farming systems aren't

This article is part of the Topical Collection: Agriculture, Food \& Covid-19.

\section{Jeff Moyer}

jeff.moyer@rodaleinstitute.org

1 Rodale Institute, Kutztown, USA aligned with what science has identified as the best foods for citizens to properly maintain health and rebuild our natural immune systems. The COVID-19 pandemic has merely pointed out a problem that has been lurking on the sidelines for decades: our medical system is overburdened attempting to treat lifestyle-related diseases with pharmaceutical intervention rather than dietary and lifestyle changes, while farmers have never thought about human or soil health as the metrics by which they are judged. The fact that our soils are being depleted of the nutrients we need to sustain our health and regenerate our immune systems, and that the way we are farming is destroying the environment and ecosystems we need to survive, is not considered in conventional production. At the same time, our modern, conventional farming systems contribute up to a quarter of global greenhouse emissions and rely on toxic inputs that threaten our health, biodiversity, clean air and water, and our soil's long-term capacity to produce food-all of which ultimately jeopardize the future of human health and all of which can be mitigated by changing the model.

Regenerative organic agriculture, on the other hand, envisions a future in which farming, healthcare, and food production practices inform a prevention and intervention-based approach to human and planetary health. Science conducted at Rodale Institute through long-term field trials shows that rather than relying on toxic chemicals to solve agricultural issues and pharmaceuticals to manage disease, a food system focused on soil health can help prevent disease (Rodale Institute 2011). By integrating our food production and healthcare systems, transitioning to a regenerative organic farming model, building in access to food that improves health rather than compromises it and emphasizing nutrition and lifestyle choices that prevent disease, we could radically change the system and take control of our health through farming.

Transitioning to a future based on regenerative organic agriculture is not without its challenges. New tools will need to be put to work, new protocols will need to be learned and new support policies will need to be put in place. But together, science, markets, and people can forge a new food production model that ensures local control and access, 
demanding that the long-term health of our soils and ecosystems be considered in the process and that our food production systems work to ensure our health. We need to encourage and support a conversation between soil scientists, medical practitioners, farmers, and food professionals that focuses on the health of the people we are charged with feeding and keeping healthy. If we take advantage of this opportunity for change and open the conversation in ways that foster innovation, with a goal of human health starting with the soil, together we will achieve a future that prioritizes health as the primary metric of agricultural success.

\section{References}

Alonso, Silvia, Paula Dominguez-Salas, and Delia Grace. 2019. The role of livestock products for nutrition in the first 1000 days of life. Animal Frontiers. https://doi.org/10.1093/af/vfz033.
Rodale Institute. 2011. The farming systems trial: Celebrating 30 years. https://mk0rodaleinstitydwux.kinstacdn.com/wp-content/uploads/ fst-30-year-report.pdf. Accessed April 21, 2020.

Slavin, Joanne L., and Beate Lloyd. 2012. Health benefits of fruits and vegetables. Advances in Nutrition 3 (4): 506-516.

Steffan, J.J., E.C. Brevik, L.C. Burgess, and A. Cerda. 2018. The effect of soil health on human health: an overview. European Journal of Soil Science 69 (1): 159-171.

Publisher's Note Springer Nature remains neutral with regard to jurisdictional claims in published maps and institutional affiliations.

Jeff Moyer is the CEO of Rodale Institute, a nonprofit research institution that has been growing the organic movement through rigorous research, farmer training, and consumer education since 1947. Learn more at RodaleInstitute.org. 